\title{
LA IMPORTANCIA DE LOS DERECHOS HUMANOS EN LA CONSOLIDACIÓN DE LA PAZ EN GUATEMALA
}

\author{
María José Torres Macho*
}

\section{DE LA DECLARACIÓN UNIVERSAL DE LOS DERECHOS HUMANOS A LOS DERECHOS HUMANOS EN LOS ACUERDOS DE PAZ DE GUATEMALA}

Cuando Kofi Annan se dirigió a la Asamblea General con motivo de la celebración del 50 aniversario de la Declaración Universal de los Derechos Humanos ${ }^{1}$, su mensaje fue claro: sin derechos humanos ni la paz ni la prosperidad pueden ser sostenibles. Mary Robinson, la Alta Comisionada para los Derechos Humanos, con ocasión de un acto en París ${ }^{2}$ puso el dedo en la llaga: la prueba de fuego para la Declaración Universal de los Derechos Humanos no está en su concepción teórica, ni en su posterior desarrollo legislativo en Convenciones y Protocolos; está en su aplicación práctica, en cómo los estados aseguran la protección de los derechos de sus ciudadanos día a día. Sin embargo, Mary Robinson, también apuntó que lo que fue una premisa en 1948, el papel esencial del Estado como aval único y como posible amenaza para el individuo, está cambiando. Como consecuencia de la globalización, hay otros actores, aparte de los Estados, que toman decisiones que afectan los derechos humanos de los ciudadanos.

Uno de los aspectos más interesantes de la reforma de las Naciones Unidas ${ }^{3}$ ha sido el énfasis puesto en el carácter central de los derechos humanos en todas las actividades del Sistema de las Naciones Unidas. La Conferencia Mundial sobre Derechos Humanos en Viena en 1993 y la Cumbre Social de Copenhague de 1995, incorporan de manera explícita los derechos humanos a las actividades del Sistema de las Naciones Unidas, particularmente desde que en 1993 se crea la Oficina del Alto Comisionado para los Derechos Humanos. Además de reiterarse los principios fundamentales que se derivan de la Declaración Universal: el carácter universal, interdependiente e indivisible de los derechos humanos, se define el desarrollo social sostenible como el resultado de la interdependencia entre el respeto a los derecho humanos, el desarrollo sostenible y la democracia. En realidad, el paradigma del desarrollo humano sostenible tiene que estar basado en una estrategia de desarrollo que incorpore todos los derechos humanos, económicos, sociales, culturales, civiles y políticos.
Las Naciones Unidas, al igual que las organizaciones no gubernamentales especializadas en derechos humanos, en particular Amnistía Internacional ${ }^{4}$, están tratando de compensar el desequilibrio tradicional que ha existido en la defensa y promoción de los derechos civiles y políticos, por un lado; y los derechos económicos, sociales y culturales, por otro. A los derechos civiles y políticos se les consideraba "más derechos" ya que podían ser tipificados en derecho interno y ser reclamados ante los tribunales. Sin embargo, los derechos sociales, culturales y económicos sólo imponen obligaciones positivas en los Estados, que no son tan susceptibles de ser reclamadas ante los tribunales. En opinión de Amnistía Internacional esta situación está basada fundamentalmente en dos malentendidos: en primer lugar, es falso argumentar que los derechos civiles y políticos no imponen obligaciones positivas en los estados, ya que los estados no sólo tienen la obligación de proteger los derechos sino también de promoverlos activamente; en segundo lugar, muchos elementos de los derechos económicos, sociales y culturales son susceptibles de ser reclamados en los tribunales. Un nuevo debate se abre al enfrentar los efectos negativos de la globalización, ya que los estados no pueden por sí solos proteger los derechos de sus ciudadanos. Para Amnistía Internacional parece absolutamente evidente que hay que poner particular atención a los derechos económicos.

Los Acuerdos de Paz en Guatemala firmados en 1996, con la moderación de las Naciones Unidas, son un ejemplo reciente de cómo un texto puede ser sensible al principio de que los derechos humanos son interdependientes e indivisibles. Los derechos humanos civiles, políticos, sociales, económicos y culturales son el eje y columna vertebral del texto de los Acuerdos. Aún más, por primera vez en unos

* Oficial Político (desde marzo 1998). División de las Américas y Europa, Departamento de Asuntos Políticos de las Naciones Unidas en Nueva York. (Los puntos de vista expresados en este artículo son los de la autora, y no necesariamente los de las Naciones Unidas).

1. SG/SM/6827-HR/4393, 10 diciembre 1998.

2. Human Rights on the Eve of the Twenty-First Century. Paris, 7 December 1998.

3. "Renewing the United Nations: A Programme for Reform" $\mathrm{A} / 51 / 950$.

4. AMNESTy InTERNATIONAL, Annual Report 1998: meeting future challenges. http://www.amnesty.org/ailib/airereport/ar98/intro2.htm. 
acuerdos de paz toman relevancia y protagonismo los derechos de las mujeres y los derechos de los pueblos indígenas, derechos normalmente invisibles en las mesas de negociación. Una de las razones fundamentales es que el proceso de negociación en Guatemala, se celebró con un conflicto interno de baja intensidad, cuyo objetivo último no fue exclusivamente el cese al fuego, la separación de las partes y el establecimiento de una institucionalidad democrática mínima; su objetivo era la transformación y modernización del Estado y de la sociedad guatemalteca. Sin embargo, llama la atención, que en el lenguaje utilizado por las partes sólo se identifican con derechos humanos tres de los doce acuerdos, dos de los cuales están esencialmente centrados en derechos civiles y políticos: el Acuerdo Global sobre Derechos Humanos, el Acuerdo sobre el Establecimiento de la Comisión de Esclarecimiento Histórico, y el Acuerdo sobre Identidad y Derechos de los Pueblos Indígenas.

El fuerte contenido de derechos humanos de la Agenda de la Paz en Guatemala ha supuesto, sin duda, un desafío para la verificación del cumplimiento de los compromisos de los acuerdos a cargo de la Misión de las Naciones Unidas para Guatemala (MINUGUA). La Misión no se ha definido como operación de mantenimiento de la paz (peacekeeping $)^{5}$, sino que se enmarca dentro de los que se consideran actuaciones del Sistema de las Naciones Unidas para promover la construcción de la paz después del conflicto (postconflict peace-building) ${ }^{6}$. MINUGUA se crea como una misión de la Asamblea General ${ }^{7}$ de verificación de los derechos humanos limitando inicialmente su mandato a la verificación del Acuerdo Global sobre Derechos Humanos ${ }^{8}$, y en aquellos aspectos de derechos humanos comprendidos en el Acuerdo sobre Identidad y Derechos de los Pueblos Indígenas. En marzo de 1997, después de la firma en diciembre de 1996 del Acuerdo de Paz Firme y Duradera, la Asamblea General solicita a MINUGUA ${ }^{9}$ que, en atención a lo dispuesto en el Acuerdo sobre Cronograma para la Implementación, Cumplimiento y la Verificación Internacional verifique el cumplimiento de la denominada agenda de la paz. La verificación incluye compromisos políticos, legislativos, sociales, económicos, agrarios, étnicos, militares y cuestiones de orden público.

Es la primera vez que las Naciones Unidas verifican el cumplimiento de compromisos que permiten el disfrute de derechos económicos, sociales y culturales de los ciudadanos de un país. MINUGUA ha de verificar e informar sobre la reforma fiscal; los techos de inversión social en el presupuesto nacional; la creación de un Estado multinacional, que asume y reconoce la condición multiétnica, plurilingüe y multicultural de la sociedad; o la mejora de los derechos de las mujeres.

Por otro lado, los Acuerdos de Paz sirvieron de marco único para la organización y definición de la primera experiencia piloto ${ }^{10}$, junto con Mozambique, de coordinación interagencial en el seno de las Naciones Unidas, sobre la base de la denominada "La Nota Estratégica de País". El marco de actuación de las Naciones Unidas en Guatemala ha asumido los cuatro pilares para la implementación de los Acuerdos de Paz: a) la reinserción y la desmovilización; b) el desarrollo humano integral; c) el desarrollo humano sostenible y; c) el fortalecimiento y la modernización de estado democrático.

\section{LOS DERECHOS HUMANOS EN LA CONSTRUC- CIÓN DE LA PAZ}

Dos años han pasado desde que se firmó en Ciudad de Guatemala el 29 de diciembre de 1996, el Acuerdo de Paz Firme y Duradera. Los Acuerdos terminaron con 36 años de conflicto interno, que según el Informe "Guatemala, nunca más" " ha supuesto 150.000 muertos, 50.000 desaparecidos, un millón de desplazados internos, 100.000 refugiados y 200.000 niños y niñas huérfanos. La Guatemala de hoy es la heredera de una larga historia de regímenes autocráticos, militarización, violaciones a los derechos humanos y discriminaciones étnicas y de género.

El proceso de paz en Guatemala ${ }^{12}$, puso fin al conflicto más antiguo de América Central y el que más tardó en concluirse, se necesitaron ocho años y tres Gobiernos para firmar la paz.

5. Entre los meses de enero a mayo de 1997 se desplegaron 155 observadores militares de las Naciones Unidas para verificar el proceso de desmovilización de la guerrilla (URNG). Ver, S/1997/432, 4 junio 1997.

6. El concepto de post-conflict peace-building ha ido tomando preeminencia, para las Naciones Unidas en los últimos años. Se trata de una serie de intervenciones después de un conflicto, concurrentes e integradas, y fundamentalmente dirigidas a la consolidación de la paz para evitar que el conflicto se reinicie. Ver A/51/950, p 120-121.

7. "Establishment of a human rights mission in Guatemala", A/48/985

8. En el Acuerdo Global sobre Derechos Humanos, sección X (Verificación Internacional por parte de Naciones Unidas), párrafo $12 \mathrm{se}$ definen los derechos que la Misión va a verificar prioritariamente: derecho a la vida, la integridad y la seguridad de la persona, a la libertad de expresión, a la libertad de movimiento y a los derechos políticos.

9. Resolución 51/198 B de 27 Marzo de 1997.

10. The Country Team in Guatemala (1998): “A Contradictory but Hopeful Juncture." Presentation of the Country Team in Guatemala of its experience on UN Reform to ECOSOC. July 14, 1998.

11. Informe Presentado por el Proyecto Interdiocesano para la Recuperación de la Memoria Histórica (REMHI). Abril 1998. Guatemala, Nunca más. Guatemala.

12. En una primera fase, en 1988 se producen las primeras discusiones entre la URNG, el gobierno y diferentes sectores de la sociedad civil en el seno de la Comisión Nacional de Reconciliación. El Acuerdo de Oslo (A/45/706-S/21931, annex) firmado en marzo de 1990 establece el formato de negociación -la conciliación nacional por parte de Monseñor Quesada Toruño, y la observación las Naciones Unidas-; así como el objetivo del proceso, darle una solución política a los problemas de la Nación. En Abril de 1991 con la firma del Acuerdo de México, se establece una agenda de 11 puntos para la negociación (para ver comentarios del Acuerdo de México: AguilerA, Gabriel. 1994: Los temas sustantivos en las propuestas de paz. FLACSO, Guatemala ). Sin embargo el período que va entre julio de 1991 y enero de 1993 se caracteriza por fuertes desavenencias entre las partes y casi ningún progreso en las negociaciones. El proceso toma otros rumbos desde que se firma el Acuerdo marco para la reanudación del proceso de negociación entre el Gobierno de Guatemala y la URNG de enero de 1994).

(A/49/61-S/1994/53, annex). Las partes solicitan al Secretario General de las Naciones Unidas que el observador en la mesa, el Sr. Jean Arnault, sea el moderador. Se posibilita además la participación de la sociedad civil, mediante el establecimiento de la Asamblea de la Sociedad Civil de la cual Monseñor Quesada Toruño será el Presidente. Esta fase final supone dos años de trabajo muy intenso hasta que en diciembre de 1996, se firma el Acuerdo de Paz Firme y Duradera (A/51/796-S/1997/114, annex II). Entre 1994 y diciembre de 1996 se firman nueve acuerdos sustantivos [Acuerdo Global sobre Derechos Humanos- A/48/928-S/1994/448,annex; Acuerdo para el Reasentamiento de las Poblaciones Desarraigadas por el Enfrentamiento Armado- A/48/954-S/1994/751, annex I; Acuerdo sobre 
Desde 1997 los avances en la implementación de la Agenda de la Paz han sido notables. La URNG se desmovilizó de manera exitosa bajo la supervisión de las Naciones Unidas. Además se desmovilizaron dos de los cuerpos más denunciados por sus constantes abusos a los derechos humanos: las denominadas Patrullas de Autodefensa Civil (PAC) y la Policía Militar. Por su parte, el Ejército de Guatemala ha reducido el $30 \%$ de sus efectivos y la nueva Policía Nacional Civil ya está en funcionamiento. Prácticamente la totalidad de la población refugiada que optó por el retorno desde los campos del Chiapas en Méjico ha sido repatriada ${ }^{13}$ por el Alto Comisionado de las Naciones Unidas para los Refugiados (ACNUR). El Congreso de la República aprobó en Octubre de 1998 un paquete de 50 reformas a la Constitución de la Repúblicà, en donde se reconoce el carácter multiétnico, plurilingüe y multicultural del Estado guatemalteco, se define el nuevo papel del ejército, la reforma del sistema de justicia y la incorporación del los derechos de los pueblos indígenas. La URNG se registró en Diciembre de 1998 como partido político ${ }^{14}$, y se presenta a las próximas elecciones formando parte de la coalición de izquierda. Después de 18 meses de trabajo, la Comisión de Esclarecimiento Histórico de las Violaciones de los Derechos Humanos presentará su informe el 25 de febrero de $1999^{15}$.

Las elecciones presidenciales que tendrán lugar en Guatemala en la segunda mitad de 1999 van a poner a prueba la elasticidad y la vocación de permanencia de los compromisos de la paz. Lamentablemente, en estos primeros meses de 1999 la agenda de la paz está viendo como sus puntos prioritarios pasan a ser secundarios en función de los intereses electoralistas de los diferentes partidos políticos.

Para tratar de ilustrar las complejidades de la transición de Guatemala desde el conflicto interno hacia la gobernabilidad democrática y la reforma del Estado y la sociedad; me he centrado en tres aspectos, que en mi opinión, pueden ejemplificar cómo los derechos humanos son vehiculares para el proceso: la impunidad, la modernización de Estado y el carácter multiétnico del Estado-Nación.

\section{a) Del Estado violador de los derechos humanos al Estado de Derecho, la impunidad es el gran desafío}

La finalización del conflicto armado interno, pasaba por reconsiderar las funciones de las Fuerzas Armadas y de los cuerpos militares y paramilitares orientados a la lucha contra insurgente ${ }^{16}$. Además de la reforma del sistema de justicia. El Informe presentado por el Proyecto Interdiocesano para la Recuperación de la Memoria Histórica (REMHI) el 24 de Abril 1998, "Guatemala, Nunca más", acusa al Ejército y a los grupos paramilitares de al menos el $80 \%$ de las violaciones a los derechos humanos en los 52.021 casos de violencia documentados en el informe a lo largo de 35 años de conflicto armado interno.

La evolución de los derechos civiles y políticos en Guatemala, según MINUGUA ${ }^{17}$, presenta una doble característica: de una lado persiste una disminución cuantitativa de las violaciones de los derechos considerados prioritarios en el Acuerdo Global; por otro la escalada de violencia e inseguridad ciudadana que se vive tanto en el interior como en la capital. El delito común ha reemplazado la violencia política manteniendo los mismos niveles de inseguridad.

Los nulos avances en el esclarecimiento del asesinato de Monseñor Gerardi Conedera, responsable del Informe REHMI dos días después de su presentación en Ciudad de Guatemala, han puesto en entredicho la capacidad real del Estado de Derecho para imponer el imperio de la ley a los grupos violentos. La reacción nacional e internacional de condena ha sido unánime, y como lo planteó Americas Watch ${ }^{18}$, sirve para recordar que Guatemala aún no ha escapado de su pasado.

Ante la incapacidad estatal para dar respuesta a las crecientes manifestaciones de violencia, dar seguridad y sancionar el delito, se ha producido un alarmante aumento del número de linchamientos como expresiones sociales de la llamada justicia por cuenta propia. Además se han constatado casos de asesinatos de presuntos delincuentes en el marco de actividades denominadas "limpieza social". El patrón de violación de los derechos humanos está variando de los abusos de los cuerpos del Estado frente a los individuos a convertirse en la falta de capacidad estatal para asegurar la protección de los derechos a los ciudadanos, así como el de investigar y sancionar a quienes los trasgreden.

Hay al menos tres consecuencias, cuanto menos preocupantes, de esta tendencia:

- el apoyo popular al mayor uso y la ampliación de los supuestos en los que se aplica la pena de muerte, en clara contradicción con lo dispuesto por la Convención Inter-Americana de los Derechos Humanos ${ }^{19}$,

- la total inacción del Estado ante la denominada justicia por cuenta propia, los linchamientos, está de hecho legitimando un fenómeno violento fuera de los cauces del estado de Derecho. La verificación

el Establecimiento de la Comisión de Esclarecimiento Histórico -A/48/954S/1994/751, annex II; Acuerdo sobre Identidad y Derechos de los Pueblos Indigenas - A/49/882-S/1995/256, annex; Acuerdo sobre Aspectos Socioeconómicos y Situación Agraria A/50/956, annex; Acuerdo sobre el Fortalecimiento del Poder Civil y la Función del Ejército en una Sociedad Democrática-A/51/410-S/1996/853; Acuerdo sobre un definitivo Cese al Fuego-, Acuerdo sobre las Bases para la Incorporación de la URNG a la legalidad, incorporación de la URNG en la vida política, y El Papel de las Fuerzas Armadas en una Sociedad Democrática, el Cese al Fuego la Desmovilización y la Verificación-] y un acuerdo de calendario para verificar el cumplimiento (Acuerdo sobre el Cronograma para la Implementación, Cumplimiento y Verificación de los Acuerdos de Paz- A/51/796S/1997/114,annex II).

13. Ver, Repatriation and Reintegration in Guatemala and Local Settlement in Mexico. 1999 Global Appeal. http//:www.unhcr.ch/fdrs/ga99/gtm.htm.

14. La inscripción de la URNG, hecho histórico para Guatemala.http//www.urng.com.

15. ReUTERS, Guatemala Truth Commission Completes Report. February 11, 1999.

16. Como resultado de la aplicación de los compromisos de los Acuerdos, el Ejército accedió a reducir sus efectivos en un $30 \%$, se desmovilizaron y se disolvieron ciertas fuerzas militares y paramilitares -las Patrullas de Autodefensa Civil (PAC)-, Los Comisionados Militares y la Policía Militar Ambulante. Además se aprueba una nueva Ley de Policía Nacional Civil (PNC).

17. MinuguA, VIII Informe de Verificación del Acuerdo Global de los Derechos Humanos. A/52/946, 15 junio 1998.

18. Human Rights WaCth, Worl Report 1999, Guatemala. http://www.hrw.org/worldreport99/americas/guatemala.html.

19. Ver, AMNESTY INTERNATIONAL: Guatemala, 10 Stepts to End Impunity and Human Rights Violations, 24 abril 1997. 
de MINUGUA ${ }^{20}$ prueba que de los 120 casos de linchamientos ocurridos entre abril de 1996 y abril de 1998, que dio lugar al asesinato de más de un centenar de personas y otras muchas lesionadas; la mayoría de los casos no fueron investigados, y en los 24 casos en los que el Ministerio Público inició la investigación, no hubo ningún resultado.

- la utilización del Ejército para funciones de seguridad ciudadana en patrullas conjuntas con la nueva PNC, según los dispuesto en el Acuerdo Gubernativo 90-96, en clara contradicción con lo dispuesto en el texto de los Acuerdos ${ }^{21}$.

El delito común ha reemplazado la violencia política manteniendo los mismos niveles de inseguridad, desafiando la capacidad de las instituciones del Estado de Derecho para cumplir su tarea fundamental de protección de los derechos de los ciudadanos.

\section{b) Los derechos económicos y sociales, el gran reto de la modernización del Estado en Guatemala ${ }^{22}$}

Las estructuras económicas y sociales del país se caracterizan por un alto grado de concentración de activos productivos, en particular la estructura de la tenencia de la tierra, la propiedad del parque industrial y del ahorro y el crédito. El nivel de escolaridad en Guatemala es sumamente bajo, el promedio es de 2.3 años llegando a ser en las zonas indígenas 1.3 años. A pesar de que Guatemala tiene una población mayoritariamente indígena en 1995 sólo el 12 \% de los establecimientos tenían en marcha un programa bilingüe. La infraestructura sanitaria en las áreas rurales es muy escasa, más de un tercio de los usuarios debe recorrer un promedio de $12 \mathrm{Km}$. ó 2 horas andando para llegar al puesto de salud más cercano. Los recursos presupuestarios del Ministerio de Salud ha representado montos menores o equivalentes al $1 \%$ del PIB en la década de los 90 . La carga tributaria en Guatemala ha tenido un valor promedio del 7.8\% del PIB, siendo la media de los países latinoamericanos $15 \%$ y más de $30 \%$ en los países europeos.

Uno de los aspectos más novedosos de los Acuerdos de Paz de Guatemala es que las metas cualitativas de los Acuerdos en política social aparecen claramente definidas con porcentajes. De esta forma se establece que el crecimiento económico sostenido del PIB debe situarse a una tasa no menor del 6\%. Se plantea que en el año 2000 el gasto público ejecutado en educación y en salud en relación al PIB se incrementará en $50 \%$ respecto al gasto ejecutado en 1995. En temas de vivienda se establece que se dedique a la política de fomento de la vivienda al menos el $1.5 \%$ del presupuesto de ingresos tributarios en 1997. En aspectos fiscales se estableció la meta del $12 \%$ para el año 2000 . Se plantea también la necesidad de que Guatemala cuente con una estrategia integral de desarrollo del área rural que facilite el acceso de los campesinos a la tierra y a otros recursos productivos, que brinde seguridad jurídica y que favorezca la resolución de conflictos.

Las reformas vinculadas a los derechos económicos y sociales son las que han experimentado los mayores retrasos, y las mayores dificultades políticas. En buena medida, porque las metas económicas y sociales de los Acuerdos abarcan muchas de las causas del conflicto, sobre todo las que están asociadas al reparto desigual de los recursos, en particular:

- La política fiscal constituye uno de los instrumentos más importantes para la movilización de los recursos internos que permitan la eficiente inversión del Estado en la agenda social de la paz (salud, educación, creación de empleo, mejora de infraestructuras). Sin embargo, la verificación de MINUGUA ${ }^{23}$ muestra que en 1988 la meta intermedia era de $10.4 \%$ y los ingresos tributarios previstos se estimaban en una cantidad no superior al $9.0 \%$. A los intentos de aumentar la carga fiscal, los sectores más reaccionarios han llegado a la movilización pública en contra de iniciativas como la Ley del Impuesto Único sobre Inmuebles. Entre los aspectos más positivos están que a finales de Diciembre de 1998, se anunciaron las discusiones para generar consensos mediante el Pacto Fiscal.

- En cuanto al acceso a la tierra, aunque ha habido avances notables, como la constitución del Fideicomiso Fondo de Tierras y la propuesta de Ley del Fondo de Tierras, los conflictos por la tierra ${ }^{24}$ se agudizaron. En 1996 el Fondo Nacional para la Paz (FONAPAZ) declaraba que había recibido cerca de dos mil denuncias por problemas de tierras, otras mil por problemas limítrofes entre comunidades. Se han producido numerosas ocupaciones de fincas privadas por parte de campesinos sin tierra, en 1996 se ocuparon 104 fincas.

Es difícil imaginarse que Guatemala pudiera ser un país competitivo en el mundo global de la economía con unos indicadores desagregados de desarrollo humano tan bajos como los que se dan en determinados departamentos del país. Sin embargo el desafío de la modernización está encontrando resistencias internas, fundamentalmente de aquellos sectores que ven amenazados sus privilegios con la ejecución de la Agenda de la Paz.

\section{c) La dimensión colectiva de los derechos humanos, los derechos indígenas dentro del estado multiétnico}

Guatemala es posiblemente el país más indígena de América, cerca de seis millones de personas de un total de once, aproximadamente un $55 \%$ de la población. En varios departamentos de altiplano occidental y nororiental la población supera el $75 \%$. La cultura maya en Guatemala se compone de veinte grupos etnolinguísticos, siendo los más importantes el k'iché, el mam, el kaqchikel y el q'eqchí. Existen además dos grupos considerados como indígenas que no pertenecen a la cultura maya, los xincas, y los grarífunas.

20. Ver nota 14.

21. Ibid.

22. Ver, UNDP (1998) : Guatemala, los contrates del Desarrollo Humano.

23. A/53/421, 28 septiembre 1998

24. Ver nota 20. 
El valor nacional del Indice de Desarrollo Humano (IDH) ${ }^{25}$ coloca a Guatemala dentro de la categoría de países con un desarrollo medio; pero cuando el IDH se mide en cada Departamento, muestra que particularmente en los departamentos con población mayoritariamente indígena los valores del desarrollo humano descienden a los niveles de los países de bajo desarrollo humano (Huehuetenango, El Quiché, Totonicapán, Sololá, San Marcos, Chimaltenango y Alta Verapaz) ${ }^{26}$.

El Acuerdo sobre Identidad y derechos de los Pueblos Indígenas, firmado el 31 de marzo de 1995, es posiblemente uno de los más innovadores, es el Acuerdo que propone nuevas formas de concebir la nación guatemalteca, sobre la base del reconocimiento de los derechos culturales, políticos económicos y sociales de los pueblos indígenas. Es digno de destacarse la activa participación de los grupos mayas en la Asamblea de la Sociedad Civil (ASC), durante el proceso de negociación. Su participación les llevó a tratar de superar sus propios conflictos y a formular una posición de consenso vis a vis los sectores no indígenas en la Asamblea, así como frente al gobierno y la URNG. La ASC ${ }^{27}$ abrió un espacio político de propuesta de los grupos indígenas, sin precedente en la historia reciente de Guatemala.

El Acuerdo reconoce la identidad de los pueblos indígenas, en particular los derechos culturales: reconocimiento de los idiomas mayas, educación plurilingüe, espiritualidad y culto maya; los derechos civiles, políticos y socioeconómicos: reactivación de las comunidades y de las autoridades indígenas al nivel local, regionalización, establecimiento de mecanismos de consulta y participación, reconocimiento del derecho consuetudinario y de las tierras comunales.

El Acuerdo sobre Identidad de los pueblos Indígenas priorizó la instalación de comisiones paritarias, con representaciones del Gobierno y de las organizaciones indígenas y otras comisiones específicas para el diseño de las reformas sustantivas en torno a temas como la educación, la oficialización de idiomas, la preservación de lugares sagrados y la temática de la tierra. Las Comisiones han sido muy activas en la presentación de propuestas. La experiencia de diálogo intercultural es la primera vez que se da en Guatemala de manera estructurada, y sin duda abre los espacios para una nueva concepción del Estado-Nación

Algunos de los desafíos de la creación de la Guatemala en transición están ligados a la maduración del diálogo intercultural y la apertura de los espacios de participación política a la tradicional exclusión de los pueblos indígenas, sin embargo el camino aún es largo:

- Desde la perspectiva política, la experiencia de participación de los grupos indígenas en el marco de la Asamblea de los Sectores Civiles, y en las comisiones paritarias se ha ido fortaleciendo y traduciendo en la maduración del movimiento maya. Sin embargo, la importancia y el peso político del movimiento indígena en los partidos políticos de Guatemala es casi inexistente.

- Desde la perspectiva jurídica, la reforma de la Constitución es esencial para incorporar los derechos indígenas establecidos en los Acuerdos. La celebración del referéndum para ratificar las reformas a la Constitución, aprobadas por el Congreso en Octubre de 1998, está previsto en la primera mitad de 1999. Las voces que en Guatemala claman por la abstención o el voto en contra de las reformas son muy numerosas.

\section{CONCLUSIONES}

Indudablemente la firma de la Paz fue sólo el inicio de un proceso complejo y aún frágil, a pesar de los avances registrados hasta la fecha. Uno de los elementos más interesantes, es que las dinámicas creadas en la negociación de la paz, y en la propia ejecución de los compromisos han servido ya para generar otros esquemas de participación política. Coincido con el planteamiento hecho en el estudio "Promise and Reality, Implementation of the Guatemalan Peace Accords" ${ }^{28}$, en donde se señalan como logros del proceso mismo, además de poner fin al conflicto, el que se haya iniciado una dinámica de cambio en las relaciones tradicionales entre los grupos de poder y los grupos históricamente marginados. Es particularmente relevante el papel que ha jugado la sociedad civil en el diseño de las propuestas de los acuerdos y en su ulterior implementación, además se ha reafirmado y consolidado el sector civil en el ejercicio del poder frente al estamento militar, y se ha reconocido por primera vez desde la Conquista el carácter mayoritariamente indígena del Estado guatemalteco.

Los Acuerdos pueden ser vistos como el marco en el que la efectiva mejora en los mecanismos de protección, garantía y promoción de los derechos humanos civiles, políticos, sociales y culturales, pueden permitir realizar tres tareas esenciales para conseguir que el proceso de construcción de la paz sea sostenible ${ }^{29}$ a) la reconciliación nacional y la lucha contra la impunidad; b) la transformación y modernización del Estado que asegure unos parámetros mínimos de gobernabilidad; y, c) la transición de las dinámicas político-sociales ancladas en esquemas cuasi-coloniales hacia esquemas más plurales y económicamente más viables.

Los Acuerdos de Paz de Guatemala son herederos de los principios fundamentales que se derivan de la Declaración Universal: el carácter universal, interdependiente e indivisible de los derechos humanos. Además, incorporan un concepto de desarrollo humano sostenible que incluye todos los derechos humanos, económicos, sociales, culturales, civiles y políticos. Desde la perspectiva de las Naciones Unidas, el proceso de Guatemala está suponiendo un doble desafío.

Por un lado, MINUGUA está realizando un ejercicio pionero en la verificación internacional del cumplimiento de compromisos vinculados a los derechos económicos, sociales y culturales; y por otro, la agenda de la paz ha servido de base para la articulación y coordinación del Sistema de las Naciones Unidas y de las Instituciones Financieras Internacionales, sin precedentes en otros procesos.

25. El IDH incluye la medición de la educación (analfabetismo y matriculación), salud (esperanza de vida) e ingreso (el PIB).

26. Ibid, pag. 14 .

27. COOK, Denise (1998): "The role of the civil society in the Guatemalan Peace Process". Internal paper, Department of Political Affairs, United Nations.

28. SPENCE, R. Day, Worby, de León-Escribano, Vickers y Lanchin (1998): "Promise and Reality, Implementation of the Guatemalan Peace Accords". En: Hemisphere Initiatives, Cambridge, Masachusets

29. Ver, PECK, Connie (1998): Sustainable Peace. Carnegie Commission in preventing Deadly Conflicts New York. 


\section{RESUMEN}

Hasta 1996 se firmaron en Guatemala doce Acuerdos de Paz, de los cuales tres versan sobre derechos humanos: El Acuerdo Global sobre Derechos Humanos, el Acuerdo sobre el Establecimiento de la Comisión de Esclarecimiento Histórico y el Acuerdo sobre Identidad y Derechos de los Pueblos Indígenas. La totalidad de los Acuerdos constituye el pilar para conseguir la desmovilización y reinserción de los guerrilleros, el desmonte de grupos paramilitares, el desarrollo humano integral, el desarrollo humano sostenible y el fortalecimiento y la modernización del Estado democrático, además de establecer el marco de acción de la Misión de las Naciones Unidas para Guatemala con el fin de verificar el cumplimiento de estos Acuerdos y promover la construcción de la paz después del conflicto.

El proceso de paz en Guatemala duró 8 años, con la firma de los Acuerdos de Paz se puso fin al conflicto más antiguo de Centroamérica, sin embargo, los notables avances conseguidos hasta ahora se enfrentan a los intereses partidarios surgidos por la proximidad de las elecciones presidenciales. Aún así, la forma de negociación de los Acuerdos de Paz ha permitido la implementación de otras modalidades de participación política, el fortalecimiento de la sociedad civil y el reconocimiento de la mayoritaria población indígena del país.

Palabras clave: Acuerdos de Paz, Derechos humanos, Naciones Unidas, Guatemala, Desarrollo Sostenible.

\section{ABSTRACT}

Until 1996, 12 peace agreements were agreed upon in Guatemala. Three of them deal with human rights: the global agreement on human rights, the agreement on the establishement of a committee for the "Esclarecimiento Historico" and the agreement on the identity and the rights of the indigenous peoples. All these agreements constitute the basis for the demobilization and reintegration into civic life of the guerrilla members, the elimination of paramilitary groupings, global human develepment, sustained development and for the strengthening and modernization of the democratic state. They also establish the framework for the reaction of the United Nations mission for Guatemala, which aims at monitoring the fulfillment of these agreemtns and promoting peace after military conflict.

The peace process lasted 8 years in Guatemala. The signature of the peace agreements put an end to the oldest military conflict in Central America. However, current achievements are meeting their limits due to party interests and pending electoral processes.

The negotiation process of the peace agreements made it possible to establish channels for political participation, the strengthening of civic society and the recognition of the majoritarian indigenous population in Guatemala.

Key words: peace agreements, human rights, United Nations.

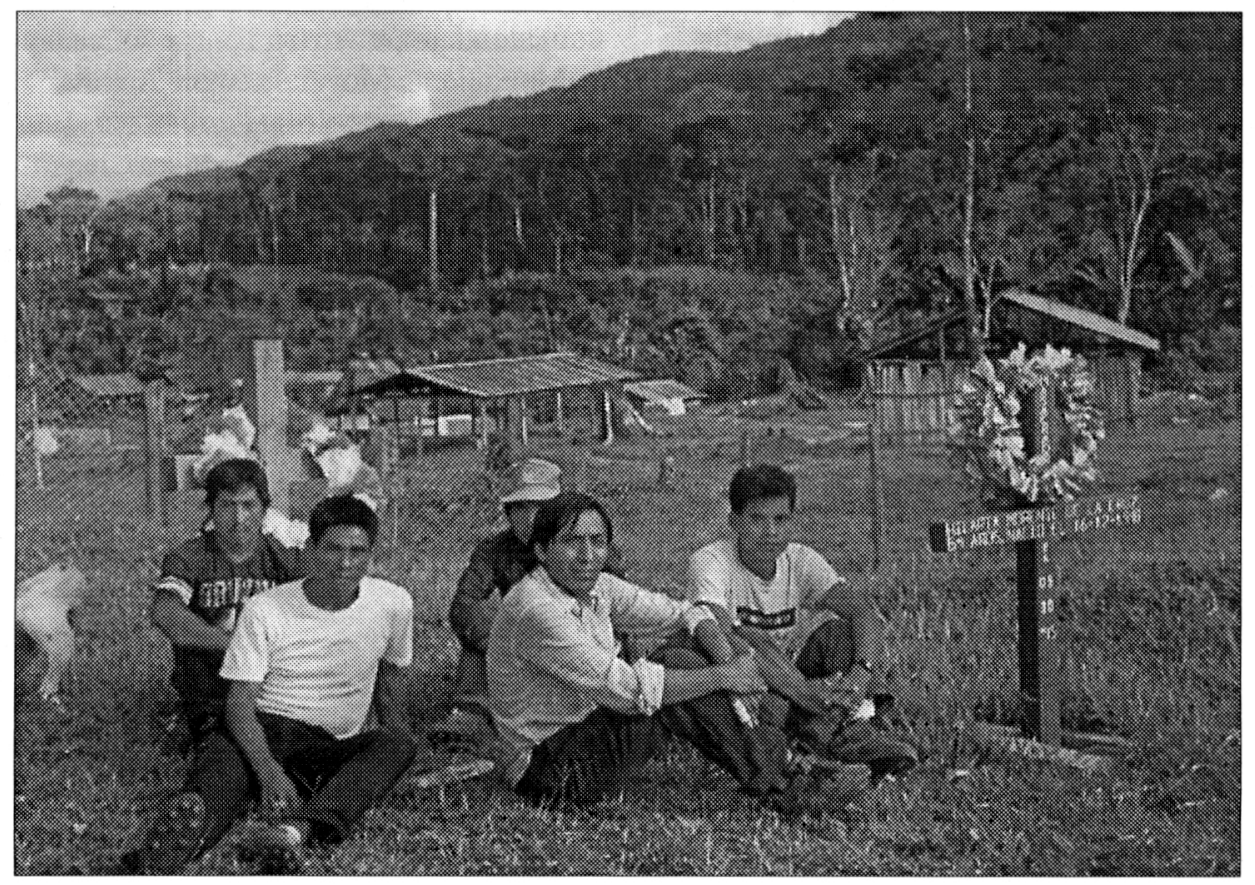

Familiares y amigos de las víctimas de la matanza de Xamán, en el lugar donde ocurrieron los hechos (César Díaz) (Gentileza de "Amnistía Internacional”) 